\title{
Estimation of Serum Interleukin- 12 (IL-12), Interleukin- 17(IL-17) and Soluble Vascular Cell Adhesion Molecule (s-VCAM) in Multiple Sclerosis
}

\author{
Omayma EL Kholy, Amira Hassouna, Ibtesam Fahmy, * \\ Reda Saad** and Radwa Taha \\ Departments of Medical Biochemistry, Neurology*, and Radiology** \\ Faculty of Medicine, Cairo University
}

\begin{abstract}
Multiple sclerosis (MS) is a complex and heterogeneous disease, and our understanding of the disease initiation mechanism and its wide clinical variability is limited. Cytokines and leukocyte endothelial adhesion play an important role in the initiation and maintenance of the inflammatory reaction in multiple sclerosis. The present study aimed to estimate the serum levels of IL-12 and IL-17 (cytokines) and sVCAM (an adhesion molecule) in different MS subtypes and to assess their relationship to disease activity, disability and MRI findings of cerebral atrophy. We analyzed serum levels of interleukins 12 and 17 (IL-12 and IL-17) and soluble vascular cell adhesion molecule (sVCAM) in 53 patients; 20 relapsing-remitting in remission (RRMS in remission), 16 relapsing-remitting in relapse (RRMS in relapse), and 17 secondary progressive (SPMS) and 15 healthy age and sex matched controls. For each patient the following were performed: clinical evaluation assessment of disability using Expanded Disability Status Scale Score (EDSS) and Magnetic resonance imaging (MRI) assessment to detect the presence of cerebral atrophy and the extent of lesion load. Results: The mean serum levels of each of studied biomarkers IL-12, IL-17 and SVCAM were elevated in all MS groups compared to the control group. Significantly higher levels were detected in SPMS versus RRMS groups (whether in relapse or RRMS in remission) and were significantly higher in $R R M S$ in relapse versus RRMS in remission. The three biomarkers were significantly correlated to each other. EDSS score showed significantly higher levels in SPMS compared to both RRMS whether in relapse or remission. However no significant difference was detected between $R R$ in relapse and in remission. EDSS correlated significantly with IL-12 and correlated weakly with each of IL-17 and SVCAM. Patients with MRI signs of cerebral atrophy showed significant higher levels of EDSS score and serum IL-12, IL-17 and SVCAM levels versus patients without cerebral atrophy. A significant correlation was found between the presence of cerebral atrophy and the EDSS score. However, no significant correlations could be detected between cerebral atrophy and the biomarkers; IL- 12, IL-17 and SVCAM. Conclusion: Serum levels of IL-12, IL-17 and SVCAM are elevated in remissionrelapse and progressive subtypes of $M S$ and in $M S$ associated with brain atrophy denoting that the inflammatory status in MS tends to persist in early and advanced stages of the disease. Immunomodulatory therapy targeting the above parameters
\end{abstract}


might seem to be beneficial to delay disease progression and/or reduces lesion activity. Except for IL-12 a rather weak relationship exists between the abovementioned markers and the degree of disability induced by MS, thus excluding their utility as reliable markers of disability.

Key words: Multiple sclerosis-interleukin -12- interleukin -17 - Vascular Cell Adhesion Molecule-cerebral atrophy.

\section{INTRODUCTION}

Multiple sclerosis (MS) is an inflammatory, demyelinating disease of the central nervous system (CNS). MS is characterized by perivenular infiltration of lymphocytes and macrophages in the parenchyma of the brain, brain stem, optic nerves, and spinal cord (1). MS is a dynamic disease, with almost constant lesion formation and a progressive clinical course leading to physical disability. The disease can present in different forms, such as primary progressive, relapsing remitting, and secondary progressie phenotypes. ${ }^{(2)}$.

Dysregulation of the blood-brain barrier (BBB) and transendothelial migration of activated leukocytes are among the earliest cerebrovascular abnormalities seen in multiple sclerosis (MS) and parallel the release of inflammatory cytokines/ chemokines. Mechanisms for breakdown of the BBB in MS are incompletely understood, but appear to involve direct effects of these cytokines/chemokines on endothelial regulation of BBB components, as well as indirect cytokine/chemokinedependent leukocyte mediated injury ${ }^{(3) .}$

The measurement of brain atrophy by magnetic resonance imaging (MRI) was reported to be sufficiently precise and accurate. It also represents one of most promising in vivo measures of neuroaxonal degeneration in MS, and it should be used extensively in the future to assess and monitor pathological evolution and treatment efficacy in this disease ${ }^{(4,5)}$. However, Miller et al. (6) reported that MRI scanning lacks precision in estimating the overall extent of BBB disruption and that correlation of the MRI scan with clinical findings and disability has been disappointing.

Developing new MS biomarkers that are sensitive and specific for BBB disruption could improve the monitoring of disease activity and response to MS therapies, and advance our understanding of dynamic MS processes participating in BBB disruption ${ }^{(7,8)}$.

Interleukin-12 (IL-12) is a proinflammatory cytokine produced primarily by blood mononuclear cells, including dendritic cells in response to antigens and mitogens ${ }^{(9)}$. It is a potent inducer of interferon-gamma (IFN- $\gamma$ ), and other Th1 cytokines that may play an important role in MS pathogenesis ${ }^{\mathbf{( 1 0 )}}$.

Interleukin-17 (IL-17) is a cytokine produced in humans almost exclusively by activated memory $\mathrm{T}$ cells, which can induce the production of proinflammatory cytokines and chemokines from parenchymal cells and macrophages ${ }^{(11)}$. Recent microarray studies of lesions of MS from patients demonstrated increased 
expression of interleukin-17, suggesting that it may be an important factor in the development of inflammatory demyelination ${ }^{(\mathbf{1 2})}$.

Endothelial activation is a key feature of multiple sclerosis pathogenesis $^{(13) .}$ Activated cerebral vascular endothelial cells express leukocyte, vascular cell, and intracellular adhesion molecules (Eselectin, intracellular adhesion molecule-1 (ICAM-1) and vascular cell adhesion molecule-1 (VCAM-1)). Adhesion molecules facilitate leukocyte adhesion to endothelium and migration into inflammatory lesions ${ }^{(\mathbf{1 4})}$. Elevations of soluble adhesion molecules have been reported as markers of $\underline{\mathrm{BBB}}$ damage. (15)

The present study aimed to estimate the serum levels of IL-12 and IL-17 (cytokines) and SVCAM (an adhesion molecule) in different MS subtypes and to assess their relationship to disease activity, disability and MRI findings of cerebral atrophy.

\section{SUBJECTS \& METHODS}

Fifty-three female patients suffering from clinically definite multiple sclerosis (MS) were enrolled in this study. They were recruited at the department of Neurology, Kasr El Aini Medical Hospital, Cairo University. Their ages ranged from 27 to 45 years.

The patients were divided according to the criteria of Lubelin and Reingold $^{(16)}$ into three groups:

- Group I: twenty patients suffering from relapsing remitting $\mathrm{MS}$, in remission (RRMS in remission);
- Group II: sixteen patients suffering from relapsing - remitting MS in relapse (RRMS in relapse); and

- Group III: seventeen patients suffering from secondary progressive MS (SPMS).

Fifteen age and sex matched healthy controls were also included in the present study. Their inclusion criteria were: no history of previous neurological symptoms, and no evidence of any inflammatory disease. Inclusion criteria of patients included: clinically definite MS (CDMS) according to criteria proposed by Poser et al. ${ }^{(17) .}$

- For relapsing - remitting patients in remission, no relapse occurred three months before the study.

- Patients in relapse were investigated within 7 days of the relapse.

- For secondary progressive patients, at least six months of continuous progression in the year before the study, with or without relapses was demanded.

The patients were previously subjected to therapeutic regimens which are accepted in Neurology department Kasr El- Aini Hospital. They were either disease specific (immunosuppressive or immunomodulatory) or symptomatic.

The patients were subjected to:

\section{A) Clinical evaluation:}

This was performed according to the neurology sheet of Neurology Department Cairo University. It included: a) History taking, b) General examination, c) Neurological examination with grading of muscle power, muscle tone and reflexes and assessment of superficial, deep and cortical sensation. 
Assessment of the clinical disability using Krutzke Multiple Sclerosis Rating Scale (Expanded Disability Status Scale "EDSS") ${ }^{(\mathbf{1 8})}$ EDSS is a method of quantifying disability in multiple sclerosis. It is a 20 step ordinal scale, which ranges between 0 (normal status) and 10 (death due to MS).The EDSS quantifies disability in eight Functional Systems (FS) and allows neurologists to assign a Functional System Score (FSS) in each of these.

\section{B) Estimation of the following} laboratory parameters :

I) Serum Interleukin-12 (IL-12): (as an immunoregulatory cytokine) by quantitative sandwich enzyme immunoassay technique: Quantikine, R \& D Systems, Inc. USA ${ }^{(19) .}$

II) Serum Interleukin- 17 (IL-17): (as an immunoregulatory cytokine) by quantitative sandwich enzyme immunoassay technique: Quantikine, R \& D Systems, Inc. USA. ${ }^{(11) .}$

III) Soluble vascular cell adhsion molecule (s-VCAM): (as a marker of endothelial dysfunction) by quantitative sandwich enzyme immunoassay technique Quantikine, R\&D Systems, Inc. USA ${ }^{(14)}$.

\section{C) Radiological assessment}

All patients selected were previously diagnosed as MS clinically and confirmed by Magnetic resonance imaging (MRI). All patients had either brain or spinal cord lesions.

Brain MRI was done within one week of blood sampling for all patients. MRI was performed using 1tesla intera (Philips) and $0.5 \mathrm{GE}$ apparatus. The MRI was done in different planes using $\mathrm{T} 1$ and $\mathrm{T} 2$ and FLAIR sequences ${ }^{(\mathbf{2 0})}$.

\section{MRI assessment included:}

1) Assessment of lesion load: for each lesion the following were determined: The site, size and lesion load. The lesion load was calculated by multiplying small lesions $(<5 \mathrm{~mm}) \times 1$, medium lesions $(5-10 \mathrm{~mm}) \quad X \quad 2$, large lesions $(>10 \mathrm{~mm}) \quad X \quad 3$, and confluent lesions X 4 . The sum of the burden is considered as the lesion load) ${ }^{\mathbf{( 2 1 )}}$.

2) Assessment of cerebral atrophy: It was detected in patients via determining relation between sulci and gyri and the relation between the width of the ventricle and the parenchymatous brain tissue.

D) Correlating serum data with MRI findings and neurological scaling scores

\section{Specimen collection}

Blood samples were collected by venipuncture in empty centrifuge tubes. Sera were separated and divided into aliquots and stored at $80^{\circ} \mathrm{C}$ till used. Haemolysed samples were discarded.

In relapsing remitting patients who were admitted to the hospital during relapse, and in secondary progressive patients, blood samples were collected on admission before starting pulse therapy (in the form of methylprednisolone, (1g) by intravenous infusion daily for 5 - 7 days during acute exacerbations, followed by oral prednisolone in a tapering schedule).

\section{Statistical Methods:}

Statistical Package for social science (SPSS) program version 9.0 
was used for analysis of data. Data was represented as mean $\pm \mathrm{SD}$. Comparing two mean values between two patients' groups was done using the student's t-test. Comparison of variables between MS subtypes and control group was determined by performing: One Way Analysis of Variance (ANOVA) followed by posthoc test. Simple linear correlation (Pearson's and Spearman correlations) were used to detect the relation between the studied variables (parametric and non - parametric, respectively) ${ }^{(22)}$

\section{RESULTS}

All patients and controls were age matched. No statistical significant difference was detected between the different studied groups concerning age $(\mathrm{p}>0.05)$

Table (1): EDSS score, total number of relapses and lesion load, in all patients' groups.

\begin{tabular}{|l|l|l|l|}
\hline \multicolumn{1}{|c|}{ Variables } & $\begin{array}{c}\text { Group I. } \\
\text { Relapsing remitting } \\
\text { in remission } \\
(\mathbf{n}=\mathbf{2 0})\end{array}$ & $\begin{array}{c}\text { Group II. } \\
\text { Relapsing remitting } \\
\text { in relapse } \\
(\mathbf{n}=\mathbf{1 6})\end{array}$ & $\begin{array}{c}\text { Group III. } \\
\text { Secondary } \\
\text { progressive } \\
(\mathbf{n}=\mathbf{1 7})\end{array}$ \\
\hline EDSS & $3.30 \pm 1.0^{\mathbf{a}}$ & $3.82 \pm 1.02^{\mathbf{a}}$ & $5.50 \pm 1.20^{\mathbf{b}}$ \\
\hline Total number of relapses & $3.85 \pm 1.56^{\mathbf{a}}$ & $8.05 \pm 2.63^{\mathbf{b}}$ & $6.25 \pm 2.40^{\mathbf{b}}$ \\
\hline Lesion load & $8.5 \pm 3.05^{\mathbf{a}}$ & $15.2 \pm 3.94^{\mathbf{b}}$ & $11.93 \pm 4.24^{\mathbf{c}}$ \\
\hline
\end{tabular}

Values were represented as means $\pm S D$

$n=$ number of subjects

$P$-value is significant if $<0.05$.

Different letters $(a, b, c)$ indicate significant difference among compared groups.

Similar letters indicate no significant difference among compared groups.

EDSS score: Expanded Disability Status Scale score

The mean value of EDSS score was significantly higher in the secondary progressive (SP) patients (group III) compared to patients with RRMS in remission (group I, $\mathrm{P}=0.0001$ ) and in relapse (group $\mathrm{I}$, $\mathrm{P}=0.0001)$. No significant difference was detected between group I (RRMS in remission) and group II (RRMS in relapse) $(\mathrm{P}>0.05)$ [Table (1)].

The mean of total number of relapses was significantly higher in both the RRMS in relapse (group II) and SPMS (group III) compared to RRMS in remission (group I)
( $\mathrm{P}=0.004$ and 0.01, respectively). However, no significant difference was detected between RRMS in relapse (group II) and SPMS (group III, $\mathrm{P}>0.05$ ) [Table (1)].

The mean values of the lesion load detected by MRI was significantly higher in the RRMS in relapse (group II) compared to each of RRMS in remission (group I) and SPMS (group III) $(\mathrm{P}=0.0001$ and $\mathrm{P}=0.01$, respectively). SPMS (group III) also showed significantly higher levels compared to RRMS in remission (group $\mathrm{I}, \mathrm{P}=0.01$ ) [Table (1)]. 
Table (2): Serum levels of IL-12, IL-17 and SVCAM in control group and the different studied groups of patients with multiple sclerosis.

\begin{tabular}{|l|l|l|l|l|}
\hline \multicolumn{1}{|c|}{ Variables } & \multicolumn{1}{|c|}{$\begin{array}{c}\text { Controls } \\
(\mathbf{n}=\mathbf{1 5})\end{array}$} & $\begin{array}{c}\text { Group I. } \\
\text { RRMS in } \\
\text { Remission } \\
(\mathbf{n}=\mathbf{2 0})\end{array}$ & $\begin{array}{c}\text { Group II. } \\
\text { RRMS in Relapse } \\
(\mathbf{n}=\mathbf{1 6})\end{array}$ & $\begin{array}{c}\text { Group III. } \\
\text { Secondary } \\
\text { progressive } \\
(\mathbf{n}=\mathbf{1 7})\end{array}$ \\
\hline $\mathbf{I L 1 2}(\mathbf{p g} / \mathbf{m L})$ & $1.50 \pm 0.51^{\mathbf{a}}$ & $3.04 \pm 0.95^{\mathbf{b}}$ & $6.53 \pm 1.0^{\mathbf{c}}$ & $11.7 \pm 3.11^{\mathbf{d}}$ \\
\hline $\mathbf{I L - 1 7}(\mathbf{( p g / m L )})$ & $2.30 \pm 0.52^{\mathbf{a}}$ & $3.75 \pm 1.4^{\mathbf{b}}$ & $8.2 . \pm 1.49^{\mathbf{c}}$ & $13.72 \pm 2.98^{\mathbf{d}}$ \\
\hline $\begin{array}{l}\mathbf{S V C A M} \\
(\mathbf{n g} / \mathbf{m L})\end{array}$ & $508.33 \pm 40.5^{\mathbf{a}}$ & $580.5 \pm 93.0^{\mathbf{b}}$ & $710.62 \pm 83.2^{\mathbf{c}}$ & $888.23 \pm 95.34^{\mathbf{d}}$ \\
\hline
\end{tabular}

Values were represented as means $\pm S D \quad n=$ number of subjects

$P$-value is significant if $P<0.05$.

Different letters $(a, b, c)$ indicate significant difference among compared groups. Similar letters indicate no significant difference among compared groups.

IL-12: Interleukin-12, IL-17: Interleukin-17; s-VCAM: Soluble vascular cell adhesion molecule.

In Table (2), the mean levels of serum IL-12, IL-17 and sVCAM in all patients' groups were significantly higher compared to the control group. The mean levels of these parameters were significantly higher in the SPMS patients (group III) compared to each of group I (RRMS in remission) and group II (RRMS in relapse) $(\mathrm{P}=0.0001$ for each). A significant difference was detected between mean levels of group II (RRMS in relapse) versus group I (RRMS in remission) $(\mathrm{P}<0.05)$ [Table $(2)]$.

- $\quad$ Significant correlations could be detected between IL-12 and each of IL-17, sVCAM (Fig.1), and EDSS score (Fig.2); $(\mathrm{r}=0.8,0.73$ and $0.57 ; \mathrm{P}=0.0001,0.0001$ and 0.001 respectively). Significant correlations could be detected between IL-17 and sVCAM ( $\mathrm{r}=$ $0.7, \mathrm{P}=0.0001$ ) (Fig. 3).

- No significant correlations could be detected between each IL-12, of IL-17, sVCAM and, total number of relapses or lesion load.

- Lesion load correlated significantly with total number of relapses ( $\mathrm{r}=0.6, \mathrm{P}=0.001)$ (Fig.4).

- MRI detected signs of cerebral atrophy in 17 patients; 4 from RRMS in remission group, 3 from RRMS in relapse group and 10 patients from SPMS group. 
Table (3): Mean levels of serum IL-12, IL-17, sVCAM, EDSS score, total number of relapses and lesion load in patients with MRI signs of cerebral atrophy versus patients without MRI signs of cerebral atrophy.

\begin{tabular}{|l|l|l|l|}
\hline & $\begin{array}{c}\text { Patients with signs of } \\
\text { cerebral atrophy } \\
(\mathbf{n = 1 7} \text { cases) }\end{array}$ & $\begin{array}{c}\text { Patients without signs of } \\
\text { cerebral atrophy } \\
(\mathbf{n}=\mathbf{3 6} \text { cases) }\end{array}$ & P value \\
\hline Serum IL-12 (pg/mL) & $9.28 \pm 4.56$ & $5.73 \pm 3.38^{*}$ & $0.004^{*}$ \\
\hline Serum IL-17 (pg/mL) & $10.01 \pm 4.45$ & $7.48 \pm 4.57^{*}$ & $0.03^{*}$ \\
\hline Serum sVCAM (ng/mL) & $805.2 \pm 205$ & $677.5 \pm 115.1^{*}$ & $0.01^{*}$ \\
\hline EDSS score & $5.17 \pm 1.46$ & $3.63 \pm 1.12$ & $0.0003^{*}$ \\
\hline Total number of relapses & $6.35 \pm 2.82$ & $5.72 \pm 2.8$ & $>0.05$ \\
\hline Lesion load by MRI & $12.0 \pm 4.9$ & $11.58 \pm 4.56$ & $>0.05$ \\
\hline
\end{tabular}

Values were represented as means $\pm S D \quad n=$ number of subjects

* indicates significant difference between compared groups $(P<0.05)$.

IL-12: Interleukin-12; IL-17:Interleukin-17; s-VCAM: Soluble vascular cell adhesion molecule. EDSS score: Expanded Disability Status Scale score

Significant higher levels were found in each of serum IL-12, IL-17, sVCAM and EDSS score (Fig 5), in patients with MRI signs of cerebral atrophy versus those without MRI signs of cerebral atrophy $\left(\mathrm{P}=0.004^{*}\right.$, $0.03 *, \quad 0.01 *$ and $0.0003 *$, respectively). However there was no statistical significant difference in the total number of relapses or in lesion load between the two patients' groups $(\mathrm{P}>0.05)$ [Table (3)].

Non-parametric correlation detected that MRI signs of cerebral atrophy were significantly correlated with EDSS score $\left(\mathrm{r}=0.51, \mathrm{P}=0.001^{*}\right)$. No significant correlations could be detected between MRI signs of cerebral atrophy and each of IL- 12, IL-17 and sVCAM. 


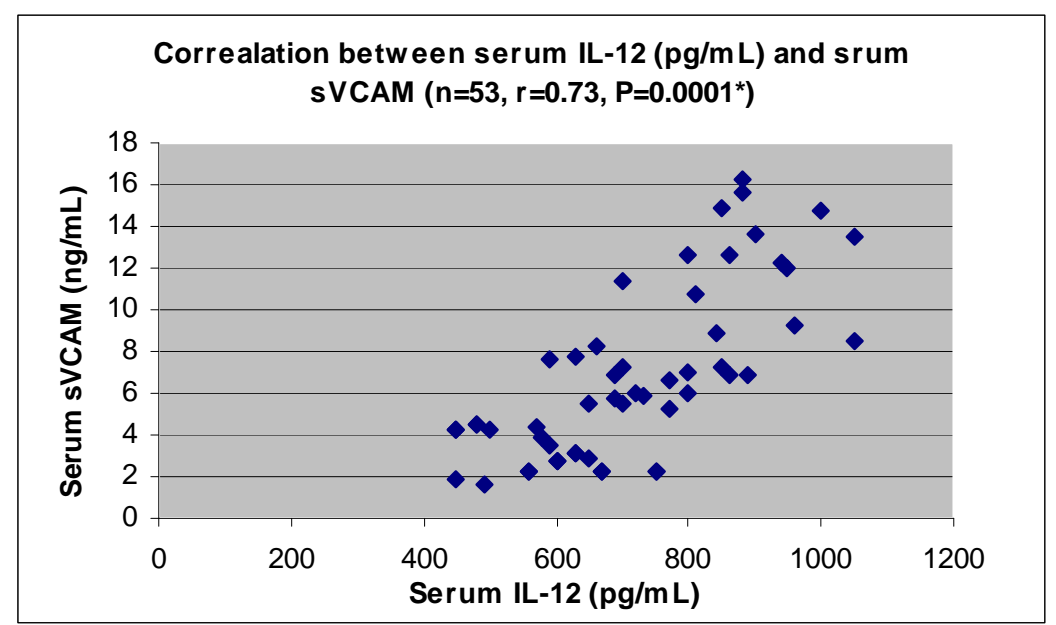

Fig (1): Scatter plot showing correlation between serum IL-12 $(\mathrm{pg} / \mathrm{mL})$ and sVCAM $(\mathrm{ng} / \mathrm{mL})$ in all multiple sclerosis patients

-IL-12: Interleukin-12

-sVCAM: Soluble vascular cell adhesion molecule

$-n=$ number of subjects

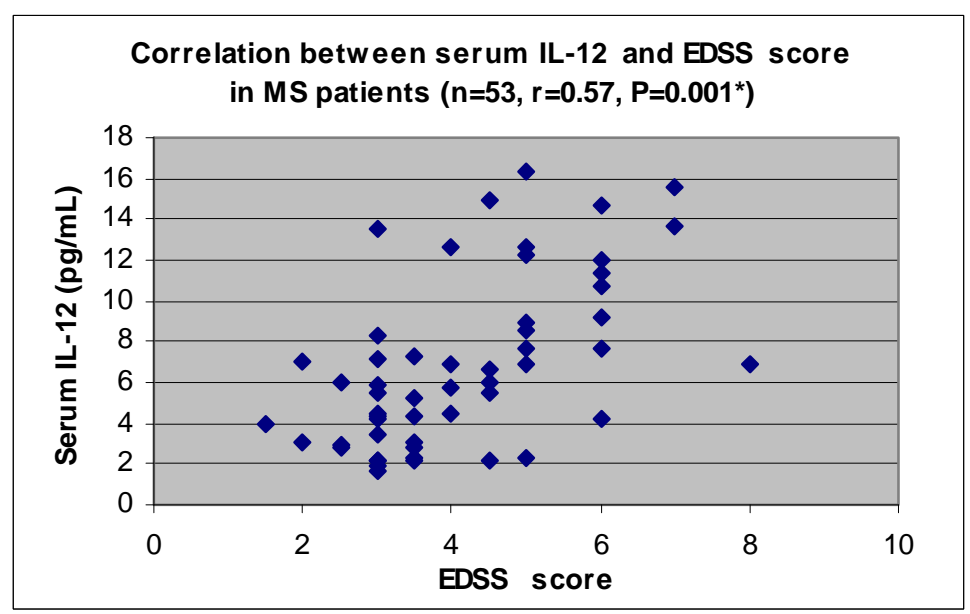

Fig (2): Scatter plot showing correlation between IL-12 (pg/mL) and EDSS score in all multiple sclerosis patients

-MS; multiple sclerosis; IL-12: Interleukin-12; EDSS score: Expanded Disability Status Scale score

$-n=$ number of subjects 


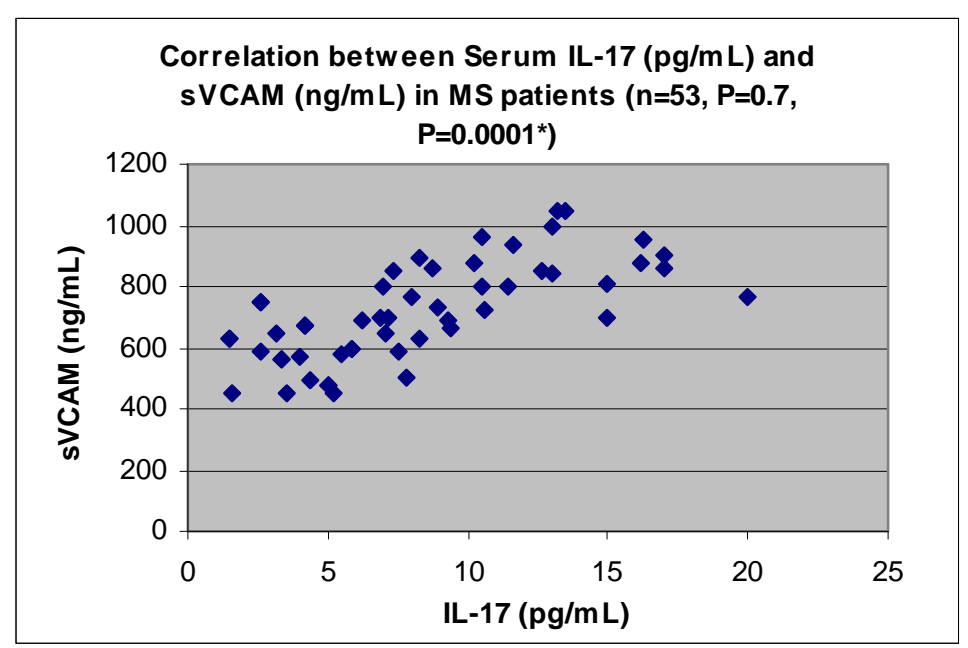

Fig (3): Scatter plot showing correlation between serum $I L-17(\mathrm{pg} / \mathrm{mL})$ and sVCAM (ng/mL) in all multiple sclerosis patients

-MS; multiple sclerosis; IL-17: Interleukin-17; sVCAM: Soluble vascular cell adhesion molecule

$-n=$ number of subjects

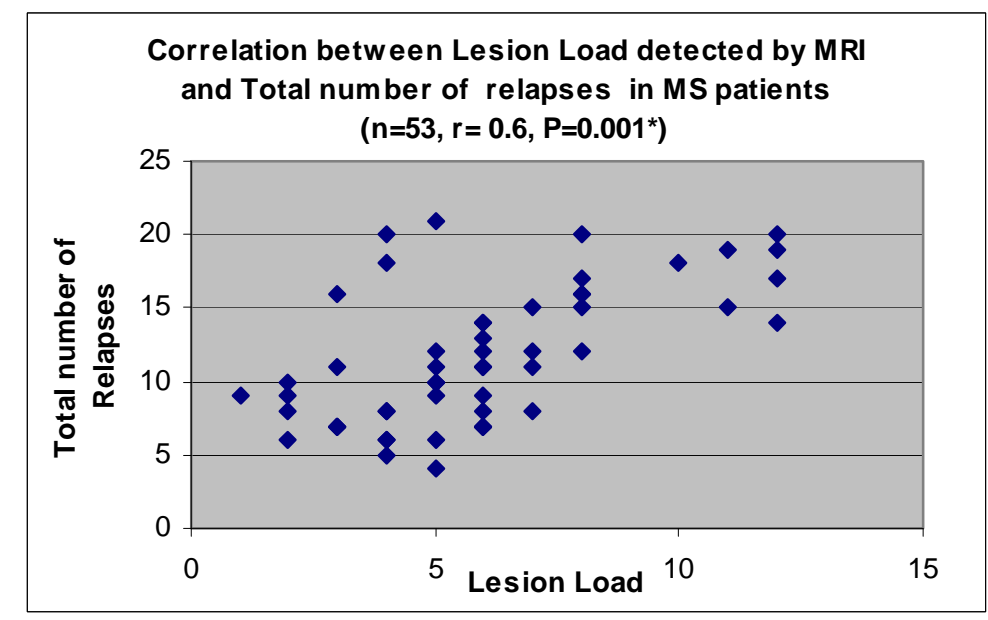

Fig (4): Scatter plot showing correlation between lesion load and total number of

-MS; multiple sclerosis relapses in all multiple sclerosis patients

-MRI: magnetic resonance imaging.

$-n=$ number of subjects 


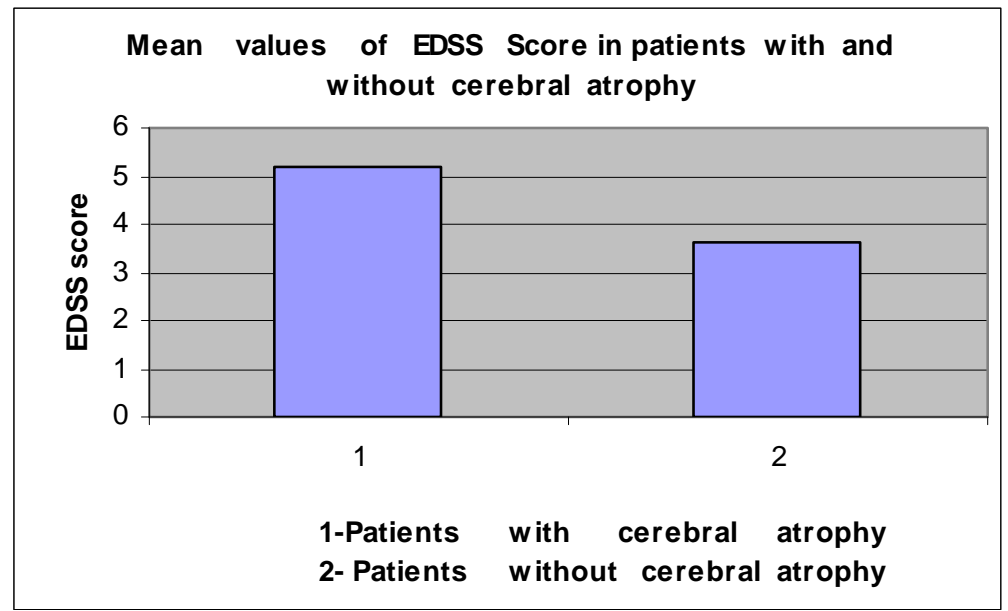

Fig (5): Mean levels of EDSS score in patients with cerebral atrophy (n=17 cases) and without cerebral atrophy ( $n=36$ cases)

-EDSS score: Expanded Disability Status Scale score

\section{DISCUSSION}

MS is postulated to be a T cellmediated autoimmune disease characterized clinically by a relapsing-remitting (RR) stage followed by a secondary progressive (SP) phase. The status of the innate immune system and its relationship to the stages of MS is not well understood. McDonnell et al. (23) provided evidence for significant immunological heterogeneity in MS and suggested that different leucocyte/endothelial cell interactions may be active in various MS subgroups.

The present study aimed to estimate the serum levels of IL-12 and IL-17 (cytokines) and sVCAM (an adhesion molecule) in different MS subtypes and to assess their relationship to disease activity, disability and MRI findings of cerebral atrophy.

In the present study the mean serum levels of each of studied biomarkers IL-12, IL-17 and sVCAM were elevated in all MS groups compared to the control group. Significantly higher levels were detected in SPMS versus RRMS groups (whether in relapse or RRMS in remission). The levels of the above biomarkers were significantly higher in RRMS in relapse versus RRMS in remission. The three biomarkers of activity or inflammation were significantly correlated to each other. Inflammation is thought to occur early during the relapsing-remitting phase of MS (RRMS), whereas in the later phases of MS such as secondary progressive MS (SPMS), inflammation tends to diminish. On the contrary, the present results 
showed that inflammatory cytokines and the adhesion molecules (IL-12, IL-17 and sVCAM) continue to be released at high levels in SPMS suggesting the coexistence of inflammation in late MS. Similar results were reported by Filion et al. (24).

IL-12 is a heterodimeric molecule composed of p35 and p40 subunits. Increased levels of IL-12 in MS whether RRMS or SPMS was

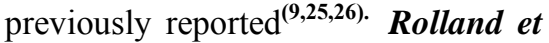
al. ${ }^{(27)}$ reported that in RRMS and in SPMS a significant increase in IL-12 and IFN- $\gamma$ production was detected. In RRMS the increase was found before relapse. Ichikawa et al. and Hart et $\boldsymbol{a l}^{\mathbf{( 2 8 , 2 9 )}}$ reported that treatment with anti-IL-12 antibodies during ongoing disease within the CNS delay disease progression and/or reduces lesion activity.

Analysis in vitro have defined IL12 as an important factor for the differentiation of naive $\mathrm{T}$ cells into $\mathrm{T}$ helper type $1 \mathrm{CD}^{+}$lymphocytes secreting interferon- $\gamma$ through activation of STAT4. IFN- $\gamma$ signals activate a downstream transcription factor, that enhances the expression of genes specific to Th1 cells ${ }^{(12,30 \text {, and }}$ 31). The mode of action of IL-12 in MS may be due to up regulation of IL-18 receptor expression. It was reported that IL-18 has an important role in cellular adhesion, being the final common pathway used by IL-1 and TNF- $\alpha$ that leads to ICAM-1 expression. Thereby, these two cytokines synergize to stimulate IFN$\gamma$ release $^{(32)}$.

Ranjbaran et al. ${ }^{(33)}$ reported that IL-12 correlated with that of IFN- $\gamma$ and IFN- $\gamma$-inducible chemokines.
Bartosik-Psujek and Stelmasiak, (34) reported that during the relapse of MS, a significant change in chemokine levels both in serum and CSF and IL-12 in CSF were noted. Stamatovic et al. ${ }^{(35)}$ suggested that in MS, chemokines (chemo-attractant cytokines) do not merely direct leukocytes to areas of injury. They also have direct and indirect effects on the BBB leading to BBB disruption, facilitating entry of leukocytes into brain, and inducing vasogenic brain edema formation. Thus IL-12 through induction of IFN- $\gamma$ and chemokine production may have an important role in the BBB disruption in MS. Chemokine inhibition may be a new therapeutic target to reduce vasogenic brain edema.

Huseby et al. ${ }^{(36)}$ suggested that the perceived central role for IL-12 in autoimmune inflammation, specifically in the brain, has been misinterpreted and that IL-23, and not IL-12, is the critical factor in this response. The cytokine IL-23, which comprises the p40 subunit of IL-12 but with a different p19 subunit, is produced predominantly by macrophages and dendritic cells and has an essential role in brain inflammation ${ }^{(37)}$. Dembinsky et al. ${ }^{(38)}$ demonstrated an abnormal Th1 bias in dendritic cells from MS patients, related to IL-23, and Chen et al. ${ }^{(39)}$ suggested that the IL-23 pathway may be a novel therapeutic target for the treatment. They demonstrated that blocking of IL-23 or its downstream factors IL-17 and IL-6, but not the IL$12 /$ IFN- $\gamma$ pathways, can significantly suppress disease development. It was reported that IL-23 induces the differentiation of naive $\mathrm{CD} 4(+) \mathrm{T}$ 
cells into highly pathogenic helper $\mathrm{T}$ cells that produce IL-17, IL-6, and TNF-alpha, but not IFN-gamma and IL-4 ${ }^{(12)}$.

IL-17 is a cytokine which is produced by activated CD4+ T cells, and was reported to synergize with IFN- $\gamma$ to enhance the production of proinflammatory cytokines including IL-6 and IL-8 ${ }^{(40)}$.

Matusevicius et al. (11) demonstrated increased numbers of IL-17 mRNA expressing mononuclear cells in MS with the highest numbers in blood during clinical exacerbations compared to remission. This is in accordance with the present study suggesting that IL-17 may be a biomarker in disease activity. Kira et $\boldsymbol{a l}^{(41)}$ also reported that serum IL-17 was markedly elevated in opticospinal multiple sclerosis.

Crawford et al $^{(42)}$ reported that MS subtypes have immunological difference; myelin-specific CD8 $+\mathrm{T}$ cells appear to be more abundant in patients with RRMS than in healthy persons or in those with SPMS. Bartosik -Psujek et al. ${ }^{(26)}$ correlated the higher activity of the disease in MS relapses with an increased activity of pro-inflammatory cytokines, while in remissions anti-inflammatory cytokines dominate. This may explain the higher levels of IL-12 and IL-17 in RRMS-in relapse compared to RRMS-in remission detected in the present study. Karni et al. ${ }^{(43)}$ suggested the abnormalities of dendritic cells in MS that might explain the immunologic basis for the different stages and clinical patterns of MS. In SPMS only Th1 (proinflammatory cytokines) is induced while in RRMS both Th1 and Th2 (anti-inflammatory cytokines) are induced. They postulated the progressive phase to be secondary to neuronal degenerative changes triggered by inflammation. This may explain the higher levels of IL-12 and -17 in SPMS versus RRMS in remission and in relapse detected in the present study.

INF- $\beta$, which has been used to treat patients with multiple sclerosis, was thought to cause a shift from a Th1-mediated to a Th2-mediated response. INF- $\beta$ has been shown to significantly increase the expression of the anti- inflammatory cytokine IL10. An important property of IL-10 is its capacity to inhibit Th1 cells. This is mediated by several mechanisms including inhibition of IL-12 production and blocking of INF- $\gamma$ synthesis by differentiated Th1 ${ }^{(44,45)}$. It is interesting that the progressive stage of MS is characterized by a loss of the ability of IL-10 to suppress INF- $\gamma$ and IL-12, as higher levels of IL-12 are detected ${ }^{(2)}$. In addition, Martin-Saavedra et al. ${ }^{(46)}$ suggested that the inhibition of nuclear DNA binding activity levels of NF-kappaB, the increase of IL-4 expression and its signaling transduction, and the decrease of IL-17 may cooperate to some of the anti-inflammatory effects of INF- $\beta$ on experimental-induced encephalomyelitis (EAE).

In an un-stimulated state, leukocytes do not readily adhere to the vascular endothelium. However, inflammatory signals induce the expression of proteins on the endothelial cell surface that promote the adhesion and extravasations of activated immune cells from the circulation into the underlying tissues. 
Key among these molecules are Pand E-selectin, intercellular adhesion molecule-1 (ICAM-1) and VCAM-1 on the endothelial cells, and their respective counter receptors, $\mathrm{P}$ selectin glycoprotein ligand-1 (PSGL1), leukocyte function-associated antigen-1 (LFA-1) and very late antigen-4 (VLA-4), on the leukocytes (47). Through interaction with its ligand, VCAM-1, the integrin, VLA4 , has been implicated in Th-1 cell migration in the animal model of MS, experimental autoimmune encephalomyelitis $^{(\mathbf{4 8})}$. The pharmacological blockade of adhesion molecules; ICAM 1, VCAM 1 and chemokines is a promising and novel therapeutic approach in $\mathrm{MS}^{(\mathbf{4 9})}$.

Regarding serum sVCAM, the present results are in accordance with others $^{(23,50,51)}$. Jackson ${ }^{(52)}$ also reported higher levels of sVCAM in SPMS than in RRMS. They added that up regulated SVCAM in blood and CSF indicate sustained potential for inflammation in the CNS throughout the clinical spectrum of MS. They suggested that some functional alteration of hematoencephalic barrier (HEB) endothelium, manifest in constant pathologic expression of sVCAM-1 molecule, is one of the pathochemical mechanisms promoting progression of MS process. This may explain the significantly higher levels of sVCAM in SPMS than in RRMS in the present study. Baraczka et al. ${ }^{(53)}$ reported that $\underline{B B B}$ damage can be assumed through higher sVCAM-1 levels. They added that clinical forms of MS differ from each other in respect to concentrations of adhesion molecules.
Giovannoni et al. ${ }^{(54)}$ found no correlation between mean levels of inflammatory markers as sVCAM-1, sICAM-1, and/or C-reactive protein (CRP)) and disease progression or changes in any of the MRI measures, disease course or relapse-rate. The difference in the MS subtypes may be attributed to the protective role of NO level. They reported that the mean levels of NO were significantly higher in patients with RRMS than in patients with SP disease. In addition raised levels of serum nitric oxide metabolites are associated with a lower number of clinical relapses and a non-progressive disease course ${ }^{(54)}$.

EDSS score showed significantly higher levels in SPMS compared to both RRMS whether in relapse or remission. However no significant difference was detected between RR in relapse and in remission. This could be attributed to the severity and progression of the course of the disease in the former subtype of MS compared with RR subtypes leading to a higher degree of disability.

EDSS correlated significantly with IL-12 and correlated weakly with each of IL-17 and SVCAM. This is in agreement with the generalized view of these cytokines and adhesion molecules as being reliable markers of inflammation or activity and not of disability. In accordance with the present results, Makhlouf et al. ${ }^{(55)}$ reported that increased IL-12 expression correlated with disability, as measured by EDSS, and also with disease activity, measured by the presence of gadolinium-enhancing magnetic resonance imaging (MRI) lesions. Rolland et al. ${ }^{(27)}$ reported that the overproduction of IL-12p40 in MS 
correlated with disease severity (EDSS) in most patients. On the contrary, Bartosik-Psujek et al. (26) found no relation between the severity of disability and the levels of IL-12, thus excluding its possible role as a prognostic factor allowing to anticipate the consequences of the relapse.

Brain atrophy is a relevant surrogate marker of the disease process in MS because it represents the net effect of various pathological processes leading to brain tissue loss. There are various approaches to quantifying central nervous system atrophy in MS. Magnetic resonance imaging (MRI) has been widely used to diagnose and monitor MS. Neurodegeneration is increasingly being recognized as an important factor in the pathogenesis of MS, and MRI measures of brain atrophy have been suggested as surrogate markers of neuroaxonal loss and disease progression. This pathology may be more relevant to the progression of disability than focal inflammation ${ }^{(56)}$.

Patients with MRI signs of cerebral atrophy in the present study showed significant higher levels of EDSS score and serum IL-12, IL-17 and sVCAM levels versus patients without cerebral atrophy, suggesting that the inflammation does not decrease in the stage of brain atrophy and/or brain atrophy may occur early in the course of MS. However no significant difference was found in total number of relapses or lesion load between the two groups. A significant correlation was found between the presence of cerebral atrophy and the EDSS score, denoting most probably that the MS-associated disability accentuates with loss of brain tissue. However, no significant correlations could be detected between cerebral atrophy and the biomarkers; IL- 12, IL-17 and sVCAM.

It is to be noted, in the present, study that ten out of the 17 patients that showed MRI signs of cerebral atrophy, were from SPMS group in which the three biomarkers showed highest levels. These results denote that cerebral atrophy and disability occur in the advanced MS subtypes.

Rudick et al. $^{(57)}$ reported that EDSS pointing from baseline and persisting at least 6 months, was associated with significantly greater brain atrophy progression. Bakshi et al. ${ }^{\text {(20) }}$ reported that brain atrophy may occur early in the course of Multiple Sclerosis and may be associated with disability. Gasperini et al. ${ }^{(58)}$ found no correlations between changes in brain volume and each of EDSS score, changes in lesion load or frequency in clinical relapses. Giovannoni et al. and Bartosik-Psujek et al. (54, 26) reported that disease progression required a sustained change in the EDSS. They found no correlation between mean levels of inflammatory markers over the study period and disease progression or changes in any of the MRI measures.

Conclusion: These results provide evidence for significant immunological heterogeneity and suggest that different leucocyte/ endothelial cell interactions may be active in various MS subtyps. Serum levels of -12, IL-17 and SVCAM are elevated in remission-relapse and progressive subtypes of MS and in MS associated with brain atrophy denoting that the inflammatory status 
in MS tends to persist in early and advanced stages of the disease. Immunomodulatory therapy targeting the above parameters might seem to be beneficial to delay disease progression and/or reduces lesion activity. Except for IL-12, a rather weak relationship exists between the above-mentioned markers and the degree of disability induced by MS, thus excluding their utility as reliable markers of disability.

\section{REFERENCES}

1 Jensen $\mathbf{J}$, Krakauer $\mathbf{M}$ and Sellebjerg F (2005): Cytokines and adhesion molecules in multiple sclerosis patients treated with interferon-beta1 $\beta$. Cytokine. 7; 29: 24-30.

2 Compslon $A$ and Coles $A$ (2002): Multiple sclerosis. Lancet 359: 1221-1231.

3 Minagar A (2003): Blood-brain barrier disruption in multiple sclerosis. Multiple Sclerosis 9: 540-549.

4 De Stefano N, Battaglini $M$ and Smith SM. (2007): [Epub ahead of print] Measuring brain atrophy in multiple sclerosis. J. Neuroimaging. 17 Suppl 1:10S$5 \mathrm{~S}$.

5 Fazekas F, Soelberg S P, Comi G and Filippi M (2007): MRI to monitor treatment efficacy in multiple sclerosis. J. Neuroimaging. 17 Suppl 1:50S$5 \mathrm{~S}$.

6 Miller JR (2000): Multiple sclerosis. In Merritt's Neurology. $10^{\text {th }}$ ed. editors. Rowland, 1. p. Lippincot, W. and Wilkins. Philadelphia: $773-7$
7 Barkhof F (2004): Assessing treatment effects on axonal loss-evidence from MRI monitored clinical trials. J. Neurol. 251 Suppl 4:IV6-12.

8 Waubant $E$ (2006): Biomarkers indicative of blood-brain barrier disruption in multiple sclerosis. Disease Markers 22: 235 - 244.

9 Losy J, Wender $M \quad G$ and Wender M (2002): Interleukin12 and interleukin-10 are affected differentially by treatment of multiple sclerosis with glatiramer acetate (Copaxone). Folia Neuropathol. 40: 173-5.

10 Rohowsky K C, Molinaro D Choudhry A, et al. (1999): Impaired InterLeukin-12 Production in MS. Mult. Scler.5:327-34.

11 Matusevicius D, Kivisakk P, He B et al. (1999): Interleukin-17 mRNA expression in blood and CSF mononuclear cells is augmented in multiple sclerosis. Mult. Scler.5:101-4.

12 Iwakura $y$ and Harumichi I $H$ (2006): The IL-23/IL-17 axis in inflammation. J. Clin. Invest. 1; 116: 1218-1222.

13 Bitsch A, Bahner, Wachter $\mathrm{C}$ et al. (2004): Interferon beta-1b modulates serum sVCAM-1 levels in primary progressive multiple sclerosis. Acta Neurologica Scandinavica. 110: 386-392.

14 Droogan AG, McMillan SA, Douglas JP and Hawkins SA (1996): Serum and cerebrospinal fluid levels of soluble adhesion molecules in multiple sclerosis: predominant intrathecal release of 
vascular cell adhesion molecule1.J.Neuroimmunol. 64:185-91.

15 Minagar A, Jy W, Jimenez JJ et al. (2001): Elevated plasma endothelial microparticles In Multiple Sclerosis. Neurol. 56: 1319-1324.

16 Lublin FD and Reingold SC (1996): Defining the clinical course of multiple sclerosis: results of an international survey. National Multiple Sclerosis Society (USA) Advisory Committee on Clinical Trials of New Agents in Multiple Sclerosis. Neurology 46 : 907-11.

17 Poser CM, Paty DW, Scheinberg L et al. (1983): New diagnostic criteria for multiple sclerosis: guidelines for research protocols. Ann. Neurol.13: 22731.

18 Petzold A, Eikelenboom MJ, Gveric D et al. (2002): Markers for different glial cell responses in multiple sclerosis: clinical and pathological correlations. Brain 125: $1462-73$.

19 Zhang M, Gately MK, Wang E et al.(1994): Interleukin-12 at the site of disease in tuberculosis. J. Clin. Invest. 93: 1733-1739.

20 Bakshi R, Minagar A, Jaisani $Z$ et al. (2005). Imaging of Multiple Sclerosis: Role in Neurotherapeutics. NeuroRx.; 2: 277-303.

21 Gawne-Cain M, O'Riordan J, Coles A et al. (1998): MRI lesion volume measurement in multiple sclerosis and its correlation with disability: a comparison of fast fluid attenuated inversion recovery and spine echo sequence. J. Neurol. Neurosurg. Psychiatry 25: 197-203.

22 Altman GA. (1991): Altman GA (ed): Practical statistics for medical research. Chapman and Hall, London.

23 McDonnell GV, McMillan SA, Douglas JP et al. (1999): Serum soluble adhesion molecules in multiple sclerosis: raised sVCAM-1, sICAM-1 and sEselectin in primary progressive disease. J. Neurol. 246: 87-92.

24 Filion LG, Graziani-Bowering $G$ et al. (2003): Monocytederived cytokines in multiple sclerosis. Clin. Exp. Immunol. 131: 324-34.

25 Mannon PJ, Ivan J., Frank Heller et al. (2004): Nonclassical CD1d-restricted NK $\mathrm{T}$ cells that produce IL-13 characterize an atypical Th2 response in ulcerative colitis. J. Clin. Invest.15; 113:1490-1497.

26 Bartosik-Psujek H, Magrys A, Montewka K M and Stelmasiak Z (2005): Change of interleukin-4 and interleukin-12 levels after therapy of multiple sclerosis relapse with methylprednisolone. Neurol. Neurochir. Pol. 39: 20712.

27 Rolland A, Jouvin-Marche E, Saresella $M$ et al.(2005): Correlation between disease severity and in vitro cytokine production mediated by MSRV (multiple sclerosis associated retroviral element) envelope protein in patients with multiple sclerosis. J. Neuroimmunol. 160: 195-203.

28 Ichikawa M, Koh CS, Inoue A et al. (2000): Anti-IL-12 antibody 
prevents the development and progression of Multiple Sclerosislike relapsing/remitting deMyelinating disease in NOD mice induced with myelin oligodendrocyte glycoprotein peptide. J. NeuroImmunol. 3: 5666.

29 Hart BA, Brok HPM, Remarque $D$ et al. (2005): Suppression of ongoing disease in a non-human primate model of Multiple Sclerosis by a humananti-human IL-12p40 antibody. J. Immunol. 175: 4761-4768.

30 Kornek B, Storch MK, Weissert $R$ et al. (2000): Multiple sclerosis and chronic autoimmune encephalomyelitis: a comparative quantitative study of axonal injury in active, inactive, and re-myelinated lesions. Am. J. Pathol. 157: 267-276.

31 Geurts JJ, Bo L, Pouwels PJ et al. (2005): Cortical lesions in multiple sclerosis: combined postmortem MR imaging and histopathology. AJNR Am. J. Neuroradiol. 26: 572-577.

32 Borish LC and Steinke JW (2003): Cytokines and chemokines. J Allergy Clin. Immunol.111: S460-S475.

33 Ranjbaran H, Sokol SI, Gallo A et al. (2007): [Epub ahead of print] An inflammatory pathway of IFN-gamma production in coronary atherosclerosis. J. Immunol. 178: 592-604.

34 Bartosik-Psujek $H$ and Stelmasiak $Z \quad$ (2005): Correlations between IL-4, IL-12 levels and CCL2, CCL5 levels in serum and cerebrospinal fluid of multiple sclerosis patients. J. Neurol. Transm. 112:797-803.

35 Stamatovic SM, Dimitrijevic OB, Keep RF and Andjelkovic AV (2006): Inflammation and brain edema: new insights into the role of chemokines and their receptors. Acta Neurochir. 96:444-50

36 Huseby ES, Liggitt D, Brabb T et al. (2001): Pathogenic role for myelin-specific CD8(+) T cells in a model for multiple sclerosis. J. Exp. Med. 194: 669-676.

37 Zhang YC, Li S, Rivera VM et al. (2004): Increased CD8+ cytotoxic $\mathrm{T}$ cell responses to myelin basic protein in multiple sclerosis. J. Immunol.172: 51205127.

38 Dembinsky AV, Balashov $\mathrm{K}$ and Weiner HL (2006): IL-23 is increased in dendritic cells in multiple sclerosis and downregulation of IL-23 by antisense oligos increases dendritic cell IL10 production. The Journal of Immunology. 176: 7768-7774.

39 Chen Y, Langrish CL, Mckenzie B et al. (2006): AntiIL-23 therapy inhibits multiple inflammatory pathways and ameliorates autoimmune encephalomyelitis. J. Clin. Invest. 116: 1317-1326. dendritic cell IL-10 production. J.Immunol. 176: 7768 - 7774.

40 Arican O, Aral M, Sasmaz S and Ciragil $P$ (2005): Serum Levels of TNF- $\alpha$, IFN- $\gamma$, IL-6, IL8, IL-12, IL-17, and IL-18 in patients with active psoriasis and correlation with disease severity. Mediators Inflamm. 5: 273-279. 
41 Kira J (2005): Multiple sclerosis. Rinsho Shinkeigaku 45: 912-5.

42 Crawford MP, Yan SX, Ortega SB et al. (2004): High prevalence of autoreactive, neuroantigenspecific CD8+ T cells in multiple sclerosis revealed by novel flow cytometric assay. Blood 103: 4222-4231.

43 Karni A, Abraham M, Monsonego A et al. (2006): Innate immunity in multiple sclerosis: myeloid dendritic cells in secondary progressive multiple sclerosis are activated and drive a proinflammatory immune response. J.Immunol. 177: 4196202.

44 Byrnes AA, McArthur JC and Karp CL (2002): Interferon- $\beta$ therapy for multiple sclerosis induces reciprocal changes in interleukin-12 and interleukin-10 production. Ann. Neurol.;51:165174.

45 Angela $J$ F Adrin $R$ and Constantinscu CS (2007): [Epub ahead of print] Reciprocal effects of INF- $\beta$ and IL-12 on STAT4 activation and cytokine induction in $\mathrm{T}$ cells. Journal of leukocyte Biology 81: 1-6.

46 Martin-Saavedra FM, Flores N, Dorado B et al. (2007): [Epub ahead of print] Beta-interferon unbalances the peripheral $\mathrm{T}$ cell proinflammatory response in experimental autoimmune encephalomyelitis. Mol. Immunol. Apr 7.

47 Derick RL, Bodary $S$ and Garovoy MR (2003): Adhesion molecules as therapeutic targets for autoimmune diseases and transplant rejection. Expert. Opin. Biol. Ther. 3: $85-95$

48 Rice GP, Hartung HP and Calabresi PA (2005): Anti-alpha 4 integrin therapy for multiple sclerosis: mechanisms and rationale. Neurology 64:1336-42.

49 Gonzalez-Amaro $R$ and Sanchez-Madrid F (2002): Intercellular adhesion molecules and chemotaxic factors in the pathogenesis of multiple sclerosis. Rev. Neurol. 35: 85-93.

50 Gusef EI, Beliaeva IA, Chekhonin VP et al. (2000): Comparative clinico-immunochemical analysis of remittent and secondary-progressive course of multiple sclerosis. Zh. Nevrol. Psikhiatr. Im S S Korsakova. 100: 51-7.

51 Elovaara I, Ukkonen $M$, Leppakynnas $M$ and Lehtimaki T. (2000): Adhesion molecules in multiple sclerosis: relation to subtypes of disease and methylprednisolone therapy. Arch Neurol. 57: 546-51

52 Jackson DY (2002): Alpha-4 integrin antagonists. Curr. Pharm. Des. 8: 1229-53.

53 Baraczka K, Nekam K, Pozsonyi $T$ et al. (2001): Concenration of soluble adhesion molecules (sVCAM-1, sICAM-1 And sL-Selectin) in cerebrospinal fluid and serum of multiple sclerosis and systemic lupus erythematosus with central nervous involvement. NeuroImmunoModulation. 9: 4954.

54 Giovannoni G, Miller DH, Losseff NA et al. (2001): Serum inflammatory markers and 
clinical/MRI markers of disease progression in multiple sclerosis. J. Neurol. 248: 431-440.

55 Makhlouf K, Weiner HL and Khoury SJ (2001): Increased percentage of IL-12+ monocytes in the blood correlates with the presence of active MRI lesions in MS. J. Neuroimmunol. 119: 1459.

56 Anderson VM, Fox NC and Miller DH (2006): Magnetic resonance imaging measures of brain atrophy in multiple sclerosis. J. Magn. Reson. Imaging. 23: 605-18.

57 Rudick RA, Bermel RA, Puli SR et al. (2005). Gray matter MRI T2 hypointensity predicts longitudinal brain atrophy in multiple sclerosis. Arch. Neurol. 62: 1371-1376.

58 Gasperini C, Paolillo A, Giugni E et al. (2000): MRI brain volume changes in relapsingremitting multiple sclerosis patients treated with interferon beta-1a. Mult. Scler. 6: 365-72. 


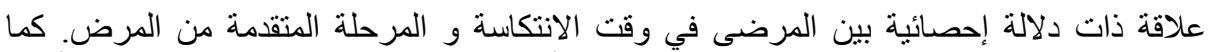

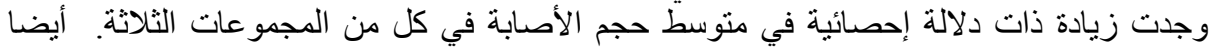

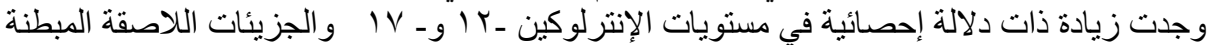

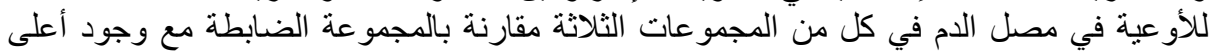

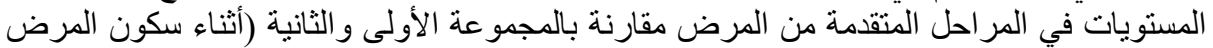

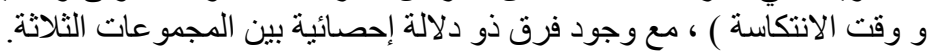

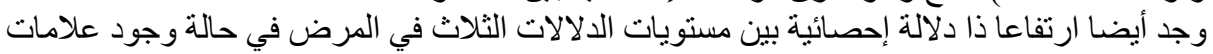

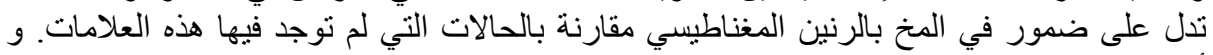

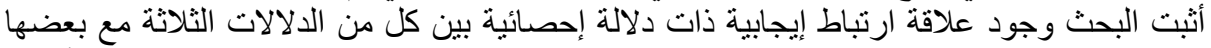

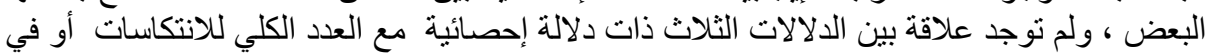

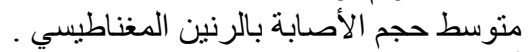

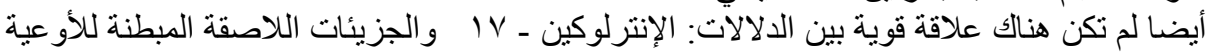

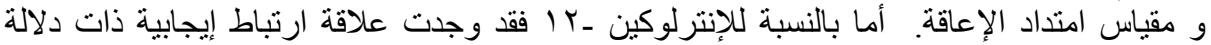

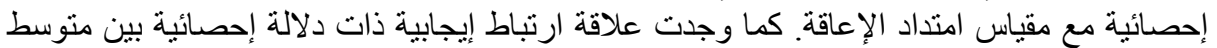

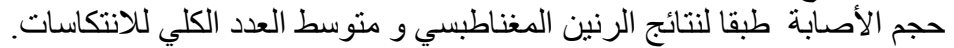

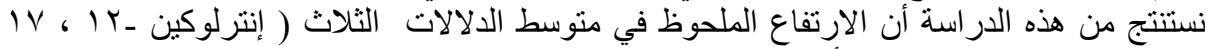

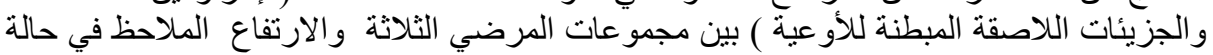

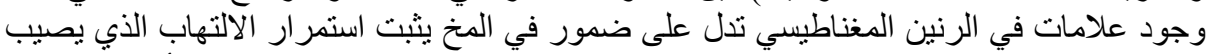

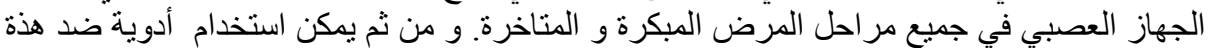

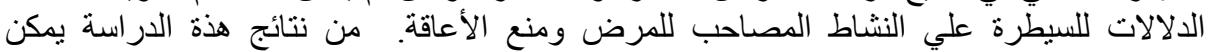
استخلاص انه يمكن استخدام هذه الدلالات لقياس نشاط المرض و ليس لقياس المتداد الإعاقة . لئل 